\title{
La generación e implementación de la estrategia en una empresa y su influencia en las políticas de calidad en la industria Ecuatoriana: un estudio de caso
}

\section{The generation and implementation of the strategy in a company and its influence on quality policies in the Ecuadorian industry: a case study}

Santiago Nájera, PhD.

Pontificia Universidad Católica del Ecuador, Ecuador

David Hernán Montenegro Moreno

Pontificia Universidad Católica del Ecuador, Ecuador

Autor para correspondencia: snajera072@ puce.edu.ec, dhmontenegro@yahoo.es

Fecha de recepción: 28 de Mayo de 2017 - Fecha de aceptación: 10 de Mayo de 2017

\section{Resumen}

La generación e implementación de la estrategia en una empresa y su influencia en las políticas de calidad constituye un tema estratégico clave para ser estudiado y el método de estudio del caso es una alternativa válida para efectuar una exhaustiva investigación cualitativa que permita obtener una comprensión significativa de un caso específico.

Palabras clave: estrategia; estudio del caso; investigación

\begin{abstract}
The generation and implementation of the strategy in a company and their influence on quality policies are a key strategic issue to be studied. And the method of study of the case is a valid alternative to carry out an exhaustive qualitative investigation that allows to obtain a significant understanding of the specific case.
\end{abstract}

Key words: strategy; study of the case; investigation 


\section{Introducción}

Una metodología apropiada para el estudio concreto de un caso único es el método de estudio del caso, el cual se halla vinculado a la investigación netamente cualitativa y tiene un enfoque orientado al alcance de una comprensión significativa del caso, a través de la realización de un exhaustivo análisis, vinculado en la presente casuística a la generación e implementación de las estrategias de diferenciación y de su influencia en las políticas de calidad de la empresa objeto de estudio.

La creación de la estrategia de una compañía constituye una representación de las respuestas de la administración a aspectos importantes tales como la concentración en un solo negocio o una diversificación (Thompson \& Strickland, 2001). La generación de la estrategia también se enfoca en el tipo de clientes que se debe elegir, ya sea un nicho de mercado amplio o un grupo reducido de clientes. Puede surgir además el tema de la búsqueda de la ventaja competitiva, por ejemplo, si se lo hiciere a través del bajo costo, de las capacidades organizacionales, o de la calidad del producto. La creación de la estrategia estará enfocada en encontrar una reacción ante las tendencias cambiantes del mercado, orientada a mantener y mejorar la competitividad empresarial para conseguir el crecimiento a largo plazo (Thompson \& Strickland, 2001).

\section{Marco Teórico}

Existen varios conceptos, teorías, relacionadas con la estrategia, la administración estratégica, la generación e implementación de la estrategia y las políticas de calidad. Todos estos aspectos se hallan relacionados entre sí en el sentido de que la generación e implementación de la estrategia constituyen el génesis de la estrategia de diferenciación para poder crear a corto y largo plazo una apropiada y sostenible ventaja competitiva, situación que derivará en una mejora de la rentabilidad y el éxito empresarial, mediante el desarrollo de productos o servicios de calidad. Adicionalmente, las políticas de calidad reciben una influencia directa de las estrategias de diferenciación y se ven reflejadas con la ventaja competitiva. Esto se plasma cuando se puede percibir la satisfacción del cliente y se evidencia un alto nivel de compromiso empresarial.

Si existiere ausencia de estrategia la organización tiene a perder competitiva lo que resulta en un desempeño inferior. Es así que, sin un marco de referencia para la toma de decisiones, ni un sentido de unión ni esfuerzos conjuntos, enfocados en reconocer el esfuerzo del equipo (Thompson A. \& Strickland A., 2001).

Para poder generar e implementar la estrategia de diferenciación es necesario enfocarse en la calidad. Por ello, es imprescindible determinar en la vida real empresarial, la manera en la cual la generación e implementación de la estrategia influye en las políticas de calidad. En la actualidad una ventaja competitiva no puede desprenderse de la calidad. (Robbins, 2010).

La percepción del valor desde el punto de vista del cliente corresponde a los beneficios recibidos y/o percibidos fruto de la adquisición del producto o servicio. Es por tanto que, la satisfacción del cliente se refiere a los sentimientos del cliente al haber realizado la compra del 
producto o servicio, es la comparación entre las expectativas y la experiencia real del cliente, conforme se observa en la figura uno.

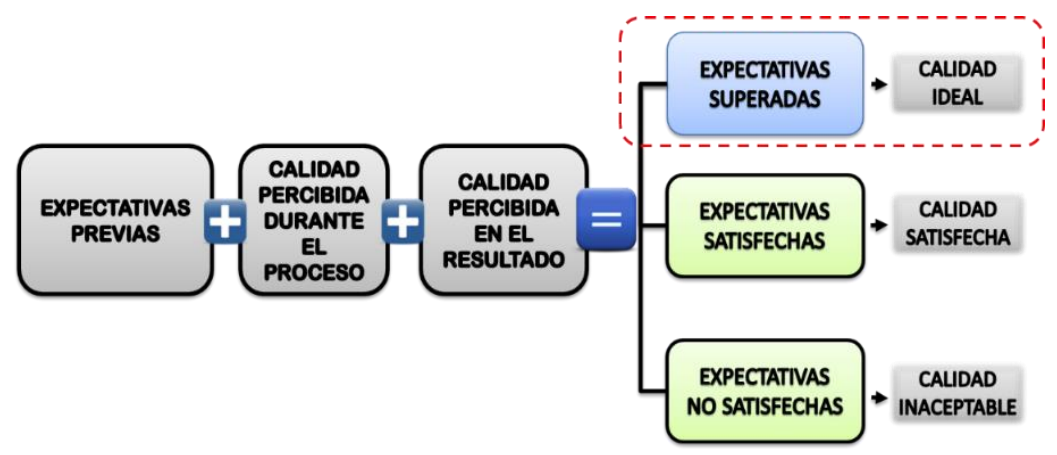

Figura 1. Calidad percibida

En este contexto, la diferencia concreta entre percepción y satisfacción constituye la recurrencia de las adquisiciones, es decir que, si el cliente ha tenido una experiencia positiva de manera general, resulta altamente probable que realice compras más frecuentes (Summers, 2006).

\section{Metodología de Investigación}

Cuando se utiliza el método de estudio del caso, una de las posibles fuentes de información a considerarse constituye la entrevista a profundidad semi-estructurada. En esta investigación corresponde la fuente primaria de información. Sin embargo, la información que se extrae de la misma corresponde a un proceso de múltiples recodificaciones de la información en búsqueda de obtener la esencia de la información obtenida, conforme se visualiza en la figura 2.
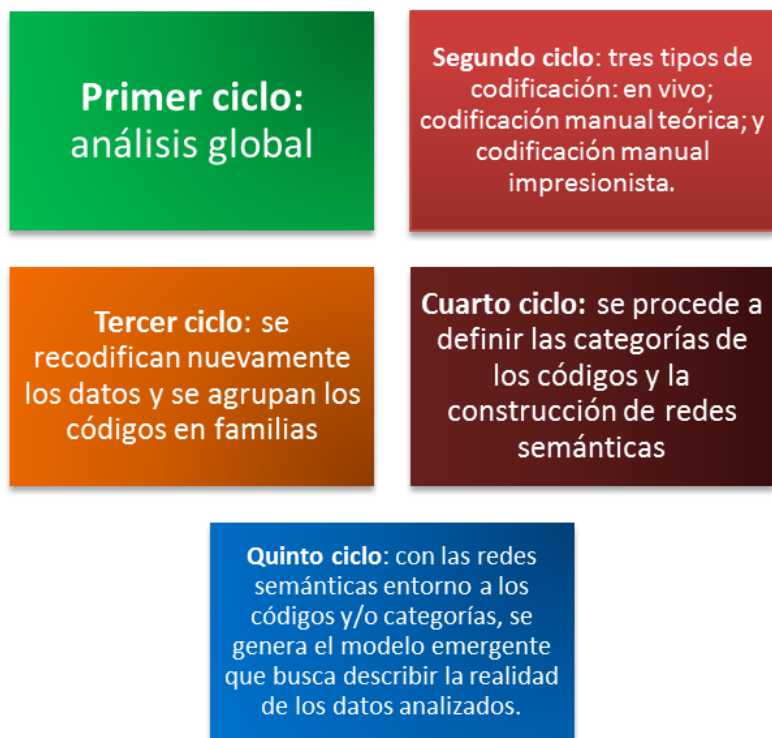

Figura 2. Proceso de codificación y recodificación.

Con la finalidad de efectuar una adecuada validación de los datos obtenidos es recomendable realizar la triangulación de datos. Esta técnica permite verificar si el trabajo de 
investigación realizado, está dentro de los parámetros correctos. La intención es que el investigador pueda asegurar la validez de los resultados de sus mediciones, contrastando con otro tipo de información, como por ejemplo reportes de ventas, manuales de calidad, declaraciones tributarias, hojas de control de producción, etc., en fin, cualquier dato que permita cotejar lo recabado en las entrevistas con información real. Adicionalmente, es importante recordar que en el estudio de casos se debe establecer un significado, no una posición; se pretende llegar a una interpretación, la cual debe ser revisada a fin de asegurar que los datos sean precisos y contribuyan a los objetivos de la investigación (Stake, 1999).

\section{Resultados}

En este estudio efectuado en una empresa textil ecuatoriana, utilizando el método de estudio del caso, se evidenció que su estrategia es enfocada fundamentalmente en las políticas de calidad, es decir en otorgar productos de calidad que satisfagan enteramente las necesidades y requerimientos de los clientes. Sus directivos entrevistados consideran que esto es factible lograrlo mediante la implementación adecuada de conceptos de calidad, buscando conseguir una ventaja competitiva que sea sostenible, en concordancia con lo que indicó Robbins (2010). Adicionalmente, se evidenció que en la empresa objeto de estudio sus directivos consideran que cuando un cliente percibe como adecuado o superior al valor entregado, la compañía podrá asegurarlo como cliente leal, lo cual mejorará el nivel de éxito de la empresa, ya que incrementarán sus ventas a corto, mediano y largo plazo. Es decir, se logran resultados financieros positivos. En este sentido, en concordancia con lo mencionado por Thompson \& Strickland (2001) con respecto de que la implementación de la estrategia mide su éxito el momento en que se obtiene una rentabilidad financiera adecuada, en la empresa objeto de estudio siempre se pretende obtener una rentabilidad que permita conseguir beneficios que sean aceptados y apreciados por los accionistas de la empresa.

\section{Conclusiones}

El método de estudio del caso es recomendable dentro de la investigación cualitativa, ya que permite verificar de manera directa la realidad de un caso específico y concreto, además de contrastar la información obtenida con la realidad. Si bien, los resultados que se obtienen de la investigación, al tratarse de un caso único, no son generalizables, sí es factible obtener una comprensión significativa del estudio efectuado estableciendo significados y explicaciones sobre el tema. Esto puede servir como base, para en un futuro efectuar estudios basados en esta metodología para varias empresas, para de este modo formar un criterio más amplio de un tema específico, es decir sentar las bases del conocimiento; como por ejemplo la generación e implementación de la estrategia en una empresa y su influencia en las políticas de calidad.

\section{Bibliografía}

Álvarez C. \& San Fabián J. (2012). La elección del estudio de caso en investigación educativa. Obtenido de http://www.ugr.es/ pwlac/G28_14Carmen_Alvarez-JoseLuis_SanFabian.html

Álvarez C., B. E. (2010). Una valoración de la estrategia, a través de la innovación, en organizaciones de Guanajuato, México. Obtenido de 
Avolio, B. (2010). Métodos Cualitativos de Investigación: Una aplicación al estudio de caso. México: Cengage Learning.

Bateman, T., \& Snell, S. (2004). Administración de una ventaja competitiva. México: Mc Graw Hill.

Deming, W. (1989). Calidad, productividad y competitividad: la salida de la crisis. México: Ediciones Díaz de Santos.

Gallardo, J. (2012). Administración estratégica de la visión a la ejecución. México: Alfaomega Grupo Editor.

Ishikawa, K. (1994). Introducción al control de la calidad. México: Diaz de Santos. 86

ISO 9001, S. G. (2008). Norma Internacional Sistema de Gestión de Calidad ISO 9001. Ginebra.

Juran, J. (2005). Critical Evaluations in Business and Management. New York: Psychology Press.

Maxwell, J. (1996). Qualitative Research Design. An Interactive Approach. London: Sage Publications.

Méndez, M., \& Reyes, A. (2004). Análisis financiero y mejoramiento de procesos productivos de una fábrica de medias, caso: Reyes Industria Textil Cia. Ltda (tesis de pregrado). Quito.

Mintzberg, H. A. (1999). Estrategía Safari:Una visita guiada por la selva de la Gestión Estrategica: Simon and Schuster. En Strategy Safari.

Patton, M. (1990). Qualitative evaluation and research methods. En K. Knafl, Designing Qualitative Studies (págs. 169-86). Beverly Hills, CA: Sage.

Phillips, L. (1998). Combining quantitative and qualitative approaches to social research in human geography, an impossible mixture? Environment and planning A, 30(2), 261-76.

Porter, M. (1980). Competitive Strategy: Techniques for Analyzing Industries and Competitors. New York.

Porter, M. (1996). What is Strategy? Harvard Business Review, November-December, 61-78.

Robbins, S. (2010). Administración. México: Pearson Education.

Stake, R. (1999). Investigación con estudio de casos. Madrid: Ediciones Morata.

Summers, D. (2006). Administración de la calidad. México: Pearson Education.

Thompson, A., \& Strickland, A. (2001). Administración Estratégica. México: Mc Graw Hill.

Yin, R. (2009). Case Study Research, Sage. E.E.U.U. 